\title{
Planejamento em Saúde e Epidemiologia no Brasil: casamento ou divórcio?
}

\author{
Luis David Castiel* \\ Francisco Javier Uribe Rivera* \\ *Escola Nacional de Saúde \\ Pública - FIOCRUZ.RJ.
}

\begin{abstract}
$O$ presente artigo discute a relaçẫo entre o instrumental epidemiológico e os métodos de planejamento das atividades de Saude Pública no Brasil. Para isso, são apresentadas, de forma resumida, concepções e atuais tendências de ambas disciplinas. Também é considerado um esquema de análise da racionalidade no processo de planejamento. Por fim, aventa razóes para explicar 0 fracasso do planejamento em saude no Brasil e o papel da epidemiologia nesta situação.
\end{abstract}

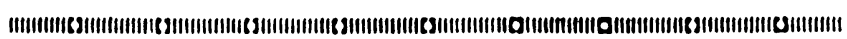

Dentre os conteúdos e disciplinas que constituem o corpo de conhecimentos do que se convencionou chamar Saúde Pública, figuram o planejamento em saúde e a epidemiologia. A princípio, pode-se considerá-los como apresentando áreas de conexão (interface), de forma que o instrumental epidemiológico viria conferir um maior grau de racionalidade técnica à elaboração e implementação dos planos e programas em Saúde Pública (Quadro 1).

Antes, porém, de abordar esta questão, é necessário discutir alguns aspectos conceituais relacionados a ambas disciplinas:

\section{CONCEPÇÕES E TENDÊNCIAS ATUAIS}

Planejamento em Saúde

Sem a preocupação em detalhar conceitos, o planejamento de saúde pode ser considerado simultaneamente como instrumento de intervenção social e como técnica -originária do planejamento econômico - de racionalização do uso de recursos escassos (dai, a necessidade de uma escala de prioridades). Tal racionalização é vista pela ótica da escolha daquelas opções al ternativas que maximizam os efeitos e barateam os custos (análise de custo/benefício).
Recebido para publicação em $0 / 08 / 85$ 
ETAPAS DO

PLANEJAMENTO

QUADRO 1

DIAGNÓSTICO

AÇÕES

EPIDEMIOLÓGICAS

- Determinação da natureza

e da magnitude do dano na população.

- Descrição da população:

- grupos vulneráveis

- distribuição

- características socioeconômico-culturais

- aspectos físicos

- acessibilidade

- açōes de saúde realizadas

- Descrição dos recursos

- Determinação das priori-

dades

- Prognóstico

\begin{tabular}{|c|c|}
\hline & $\begin{array}{l}\text { dades } \\
\text { - Prognóstico }\end{array}$ \\
\hline $\begin{array}{l}\text { DECISÃO } \\
\text { (Adoção de normas técnicas } \\
\text { e administrativas, decidindo } \\
\text { entre planos alternativos e } \\
\text { tomando em conta os condi- } \\
\text { cionamentos políticos). }\end{array}$ & - Definição de parâmetros \\
\hline \multicolumn{2}{|l|}{ PROGRAMAÇĀO } \\
\hline \multicolumn{2}{|l|}{$\begin{array}{l}\text { Objetivos - metas - fun- } \\
\text { çōes - estruturas - recursos } \\
\text { físicos - pessoal - financia- } \\
\text { mento. }\end{array}$} \\
\hline EXLCUÇĀO & $\begin{array}{l}\text { - Acompanhamento (monı- } \\
\text { toração) }\end{array}$ \\
\hline AV ALIAÇĀO & - Avaliação. \\
\hline
\end{tabular}

IONTE: MAZZAFERO, V I.. - "Uso de la Epidemiologia en la Planificación de los Servicios de Salud". In "Usos y Perspectivas de la Epidemiologia". Documento 11 do "Seminario sobre Usos y Perspectivas de la Epıdemiologıa" Publ. nọ PNSP, 84-47, OPAS. 1984 (abreviado).

O planejamento de saúde (da mesma forma que o econômico) teria, então, como características básicas:

a) "ser vol tado para o futuro;

b) visar objetivos determinados;

c) implicar a existência de um sujeito;

d) implicar a escolha de caminhos ou ações alternativas;

e) as ações necessárias para atingir os objetivos têm uma seqüência lógica, são empreendidas de modo organizado ${ }^{15}$ ". Knox et $\mathrm{al}^{y}$, em poucas palavras, considera o ponto focal do planejamento como $o$ ato de escolher entre meios alternativos visando a fins desejados (item "d"). Whittaker Ferreira ${ }^{7}$ diz que o planejamento é o contrário da improvisação.

O pressuposto da existência de um sujeito (item "c") se refere ao papel preponderante do Estado na formulação e implementação de planos e programas. Isto condiciona os resultados finais do processo no sentido de beneficiar inte- 
resses parciais de uma dada sociedade (todo planejamento é um ato de parcialidade social).

O planejamento teria, globalmente, uma dupla dimensão indissociável: política e técnica. O processo de planejamento, no entanto, é usualmente relacionado à noção de racionalidade técnica (reducionismo tecnocrático). Esta concepção percorreria todas as fases do processo: a análise da situação; a determinação de prioridades; formulação de metas, objetivos e estratégias para resolver os problemas diagnosticados e a execução e avaliação dos resultados obtidos.

Em linhas gerais, são considerados dois modelos básicos de metodologia de planejamento de saúde: 1) baseado na população, ou nas necessidades de saúde; 2) baseado na oferta de serviços. Ambos modelos se incluem no enfoque normativo do planejamento que pode ser definido como uma abordagem que omite a análise de viabilidade (política e social) na formulação das estratégias possíveis. Os modelos citados se baseiam, a princípio, na utilização de dados estatísticos e de normas de eficiência, eficácia e efetividade dos serviços (dando ênfase à informação estratégica, qualitativa, relacionada com a dinâmica social).

O modelo de planejamento a partir da população obedeceria, segundo Tannen ${ }^{22}$, à seguinte lógica:

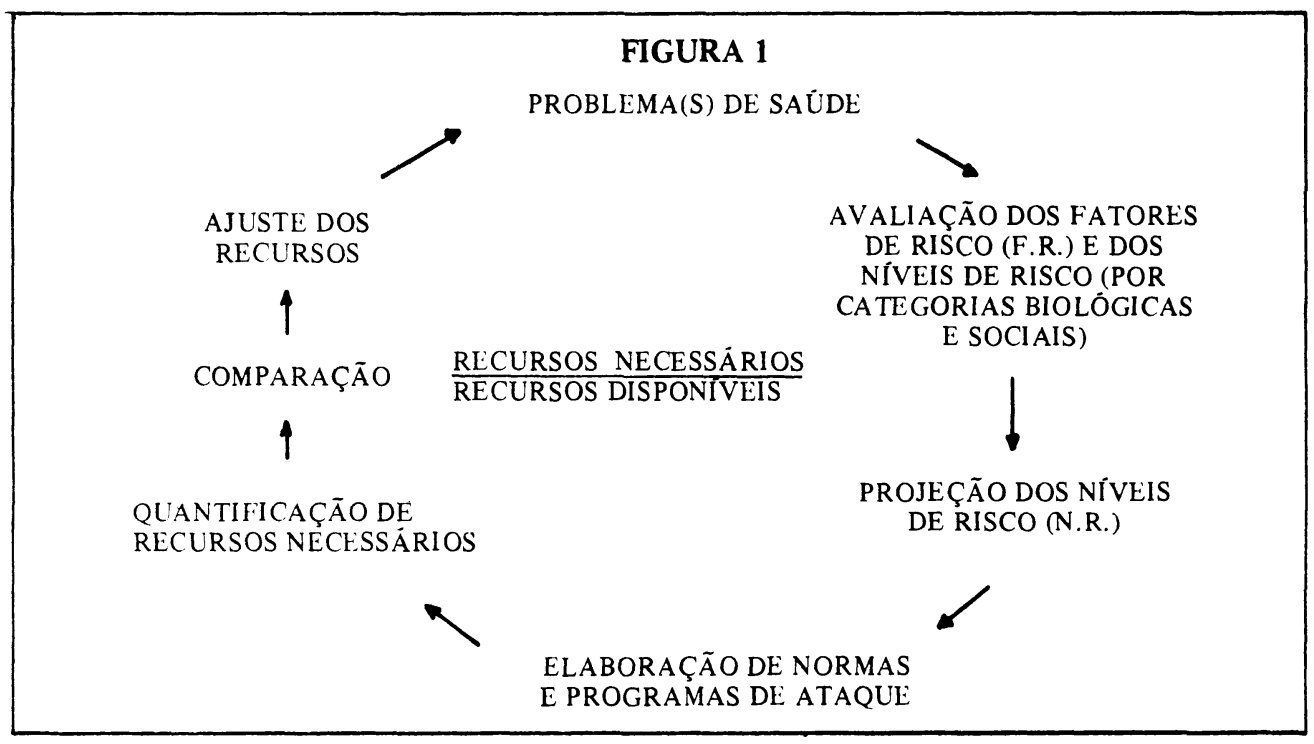

Este enfoque possibilita a aplicação da metodologia epidemiológica no que diz respeito à determinação das necessidades, utilizando-se critérios demográficos e de morbimortalidade. Abre a possibilidade, por outro lado, de se trabalhar com variáveis de caráter social, dentro da concepção 
do risco social (enfoque mais amplo de risco). E propicia, portanto, a introdução de um enfoque mais estratégico do planejamento, na medida em que a fase de projeção da situação atual dos Fatores de Risco e dos Níveis de Risco pressupõe a previsão dos cenários político e econômico. Trata-se, na sua acepção mais ampla, de uma visão que viabiliza, a priori, um tipo de intervenção intra e extra-setorial.

O modelo de planejamento a partir dos recursos obedeceria, segundo Tannen ${ }^{22}$, à seguinte lógica:

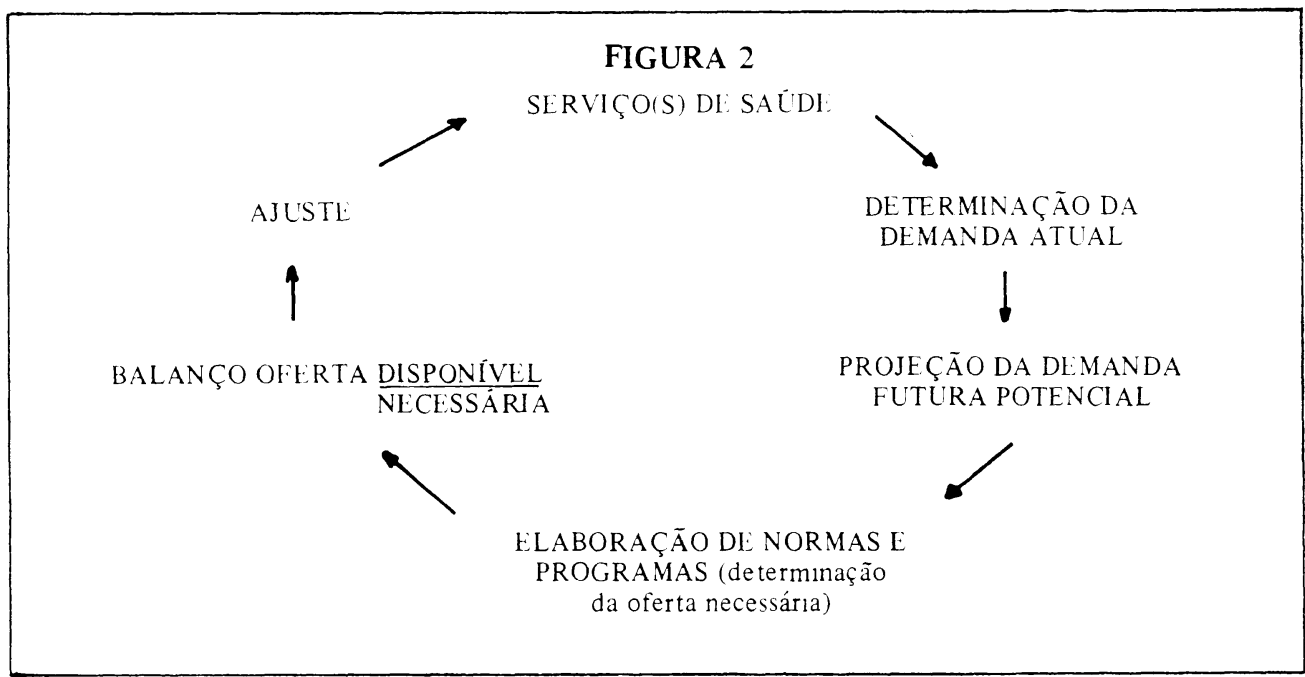

Neste caso, o ponto de partida é o serviço (que apresenta problemas de sub ou de superutilização) e a metodologia se reduz à adequação da oferta à demanda potencial (pelo uso de normas de serviço que são, às vezes, copiadas de situações-modelo). Este tipo de enfoque escamoteia a informação demográfica e epidemiológica e se limita a uma mera adequação da situação existente em termos do padrão de organização da oferta (enfoque reprodutivo).

Afirmou-se que ambos modelos podem ser encarados co. mo fazendo parte do enfoque normativo, caso essa metodologia não incorpore a dimensão estratégica do planejamento. Neste caso, a ênfase é dada à análise do(s) grau(s) de aceitação e rejeição das diretrizes programáticas por parte dos diferentes segmentos da população, dos mecanismos de representação e cooptação desses segmentos (análise de viabilidade) e a elaboração da estratégia, com base no dimensionamento dos tempos políticos mais adequados para a colocação (e grau de intensidade da colocação) do elenco de ações interligadas no conjunto programático. Tal dimensionamento implica uma permanente análise de conjuntura e previsão do cenário político-social. 


\section{EPIDEMIOLOGIA}

A epidemiologia é considerada uma ciência aplicada. Defini-la, no entanto, pode ser tarefa árdua. Lilienfeld ${ }^{13}$ listou 23 definições de epidemiologia, em ordem cronológica, a partir de 1931 até 1978 , destacando a transição da ênfase do estudo das doenças transmissíveis para o estudo das enfermidades crônico-degenerativas e outros danos à saúde (acidentes, violências). Inclusive, vale lembrar que este fato é ilustrado por Terris ${ }^{23}$ ao referir-se à segunda revolução epidemiológica*.

Para não nos estendermos numa discussão certamente supérflua, registramos a definição apresentada pelo Dicionário de Epidemiologia elaborado sob os auspícios da Associação Internaconal de Epidemiologia, em 1983: $O$ estudo da distribuição e dos determinantes dos estados e eventos relacionados à Saúde em populações e à aplicação deste estudo ao controle dos problemas de saúde ${ }^{10}$.

$\mathrm{Na}$ obra clássica de Morris ${ }^{17}$, são detalhados os usos da epidemiologia, a saber: Realização de estudos históricos da doença, diagnóstico de saúde da comunidade, avaliação do funcionamento dos serviços de saúde, estabelecimento de riscos e probabilidades individuais de sofrer agravos à saúde, identificação de sindromes, complementação do quadro clínico, busca das causas da doença. E, desde a época da sua primeira edição (1957), não parece ter havido modificações substanciais quanto à utilização dos métodos epidemiológicos.

Entretanto, têm ocorrido mudanças no pólo teórico das investigações epidemiológicas. Sobretudo a partir da década de 70, vem sendo discutida, na América Latina, uma outra abordagem na epidemiologia, preocupada com a determinação social da doença $a^{2}, 3,5,11,21$. Este modelo de causalidade é uma tentativa de superar o referencial teórico-positivista da epidemiologia clássica ou convencional, representada pela linha desenvolvida nos países centrais. Nesta, a causalidade é vista pelo ângulo da história natural da doença e das redes de multicausalidade ${ }^{12,14}, 20$. Então, dentro de tal modelo de intervenção na Saúde Pública, as ações são baseadas nos niveis de prevenção (pré-patogênico, pré-clínico, clínico) $)^{12}$.

Uma tentativa de aperfeiçoar o esquema dos níveis de prevenção foi formulada por Dever ${ }^{6}$, ao desenvolver um modelo de análise onde os fatores responsáveis pela doença são divididos em quatro, a saber: a) a organização da atenção médica;b) o estilo de vida;c) o meio-ambiente; d) a biologia humana. A análise destes quatro fatores em relação às principais causas de óbito no Canadá coloca o estilo de vida como o elemento com o maior peso na grande maioria das causas de morte. Com isto, ficaria justificada a alocação
* Já que a primeira teria ocorrido mediante o controle das doenças transmisstveis (nos paises do chamado 19 mundo).

Atualmente, esta concepção talvez devesse ser revista, uma vez que situaçóes como a doença dos legionários, herpes, SIDA (Sindrome de Imunodeficiência Adquirida) não sugerem um controle das doenças transmissiveis nestes paises. 
* SZKLO, M. - Palestra proferida no seminário sobre ensino $e$ pesquisa da epidemiologia, ABRASCO, setembro, 1984. proporcionalmente maior de recursos para medidas de educação visando a modificar estilos de vida perniciosos. No entanto, esta formulação sugere o mecanismo de culpar a vitima por esta ser obrigada, socialmente, na verdade, a adotar estilos de vida nocivos, tais como: o hábito do tabagismo, dietas ricas em lipídios saturados, etc.

Ao abordar o modelo dos níveis de prevenção, Breilh ${ }^{2}$ critica McMahon ${ }^{14}$ por dar maior hierarquia a variáveis dependentes de procéssos físicos e biológicos (idade, sexo, raça, geografia etc.), concluindo que este autor prescinde do econômico-estrutural e assimila o processo saúde-doença ao natural. O que McMahon ${ }^{14}$ faz (e, via de regra, os autores clássicos) é reduzir a estrutura econômico-social à mesma dimensão das variáveis dependentes de processos físicos e biológicos. Assim, neutralizaria seu papel determinante nas análises de causalidade das pesquisas epidemiológicas ${ }^{13}$.

A epidemiologia social procura modificar esta abordagem ao redimensionar o papel da estrutura social como fator determinante da doença (e, conforme Breilh ${ }^{2}$, gerador do que ele denomina perfis epidemiológicos de classe, caracterizando um padrão de morbi-mortalidade vinculado à classe social que o segmento da população pertence). Neste caso, a intervenção na Saúde Pública deve ocorrer, em última análise, através de mudanças na formação sócio-econômica patogênica, mediante não só medidas de saúde, mas, principalmente, por meio de ações de caráter político (como se pode notar, tal abordagem apresenta pontos em comum com o enfoque estratégico do planejamento).

Todavia, eventualmente, é mencionada a fragilidade metodológica da epidemiologia social*. Tal crítica se baseia no fato das investigações desta corrente utilizarem, via de regra, estudos ecológicos para corroborarem suas hipóteses. Devido aos problemas inerentes a este tipo de desenho, as inferências daí resultantes podem sofrer os efeitos da chamada falácia ecológica, entre outros possíveis problemas ${ }^{16}$, limitando os achados obtidos. Szklo denomina tais tipos de estudos de modelagem, por, em sua opinião, partirem de pressupostos ideológicos e não de hipóteses que possam ou não ser refutadas. Isto, entretanto, não invalida os esforços realizados dentro desta linha de pesquisa. É possível avançar por meio do aperfeiçoamento metodológico e da percepção de que existem situações em que as regularidades que se tenciona observar podem não ser alcançadas por determinados desenhos de estudos epidemiológicos.

Vale salientar o relativamente recente surgimento da Epidemiologia Clínica. Nesta orientação, os métodos epidemiológicos (com especial ênfase para os desenhos experimentais) são empregados para avaliar a adequação dos procedimentos de diagnóstico clínico (incluindo exame clínico, se- 
leção de testes diagnósticos, interpretação de dados diagnósticos) e do tratamento (incluindo prognóstico, a decisão quanto à melhor terapêtica, o grau de aderência (compliance) do tratamento e a ocorrência de efeitos colaterais) ${ }^{19}$.

Almeida Filho' critica esta vertente porque “. . . propõe simplesmente a negação do social, com o recolhimento da Epidemiologia à sua origem, pretensamente clínica, isolando artificialmente os processos saúde/doença dos processos sociais mais amplos". Além disso, a Epidemiologia Clinica parece, a principio, negar a necessidade de uma dicussão das bases teórico-conceituais da disciplina, uma vez que admite a valência do modelo clinico-experimental com fundamento epistemológico formal ${ }^{1}$. Não obstante, cabe ressaltar que a epidemiologia clínica pode sevir como instrumento de avaligação da efetividade das atividades clínicas, em crítica situação atualmente em nosso meio.

\section{A RACIONALIDADE DO DIVÓRCIO E DO FRACASSO DO PLANEJAMENTO DE SAÚDE NO BRASIL}

Giordani $^{8}$ desenvolveu um esquema de análise para o planejamento social, em que identifica três acepções para o conceito de racionalidade. De forma resumida, o autor citado considera:

19) A existência de uma acepção que tenta expressar a existência de leis de organização e funcionamento da sociedade como um todo (. .). Tal acepção se refere a um conceito abstrato tal como modo de produção capitalista ou a uma formação como a França (... .).

20) Uma noção de racionalidade parcial, ao nível das forças sociais. Pelo fato de a sociedade ser dividida em classes, a classe hegemônica procura impor seu projeto social (e sua racionalidade) ao resto da sociedade. Como diz Giordani ${ }^{8}$, "\%. .) com isto se determina o destinatário favorecido de seu uso instrumental" (...). As funções que cumpre o planejamento em uma sociedade bem determinada vão estar condicionadas pelo papel do Estado em relação aos grupos sociais atuantes pela orientação do benefício para cada um deles, pela relação que a própria atividade tenha com as outras atividades do Estado e também pelo significado do planejamento dentro das leis gerais de funcionamento de uma sociedade específica. (. .).

39) Racionalidade técnica - que se origina a partir de uma relação de eficácia entre objetivos e meios, mas que deve ser localizada num tempo e espaço determinados. Essa eficácia é alcançada por grupos ou forças sociais atuando sob determinadas formas de comportamento numa formação econômico-social bem pré-fixada. 
0 esquema de análise proposto por Giordanii ${ }^{8}$ será utilizado na tentativa de situar o papel da Epidemiologia no planejamento em Saúde no Brasil, bem como explicar o fracasso deste processo.

$\mathrm{Na}$ realidade, o planejamento em saúde segue uma racionalidade técnica incompleta. Isto porque ocorre o uso predominante do modelo a partir da oferta de serviços, com todas as suas limitações. $O$ diagnóstico (baseado na oferta ou nas necessidades) é construído e interpretado por intermédio de dados e informações conforme a escala de valores que tipificam o sistema social e os serviços de saude ${ }^{4}$. Assim, o tipo, a coleta e a análise dos dados epidemiológicos são influenciados pela racionalidade do projeto social do grupo hegemônico (na segunda acepção de Giordani ${ }^{8}$ ). E, na formação social brasileira, tal grupo não está, primordialmente, interessado em verificar as necessidades de saúde da população. Parafraseando Whittaker Ferreira ${ }^{7}$, o planejamento procura na verdade conter a pressão das necessidades em saide. Nesta perspectiva, nota-se que a metodologia epidemiológica é utilizada em determinadas atividades de Vigilância Epidemiológica. Ainda assim, o recorte das necessidades é convencional, sem que sejam focalizados grupos populacionais de maior risco de adoecer (no denominador das taxas) e, portanto, potenciais recipientes de ações de saúde. Além disso, mesmo dentro de uma ótica convencional, esta abordagem é bastante limitada pela precariedade dos sistemas de informação.

Em suma, a percepção técnica das necessidades está muito distante das necessidades sentidas ou percebidas pela população. Isto pode ser explicado, a princípio, pela flagrante escassez de recursos, não apenas como conseqüência da crítica situação econômica, mas também pela usual retração das inversões na área social e pela utilização inadequada dos recursos dentro do Setor Saúde.

Nesta perspectiva, a oferta de seviços está condicionada por interesses empresariais e lucrativos. Busca-se satisfazer às necessidades dos indivíduos enquanto agentes de consumo da tecnologia médica, serviços privados de atenção médica e de medicamentos. Não interessa mudar este quadro, ao adequar a oferta à população, mas reciclar um sistema organizado segundo as leis do mercado capitalista, donde o melhor método seja ainda o de planejamento a partir dos recursos.

Os interesses políticos e econômicos se sobrepõem aos argumentos técnicos, e nem mesmo a proposta de racionalidade técnica se completa. Pois a alocação dos recursos não obedece a critérios de eficiência, efetividade e eqüidade.

Diante desta conjuntura, a epidemiologia enquanto instrumento convencional não funcionará, pois as informações 
de saúde da população (mesmo com as limitações citadas) não refletem, usualmente, as necessidades em saúde, mas as necessidades dos serviços. $\mathrm{E}$, ainda, tendem, em geral, a serem usadas de forma parcial, já que o planejamento das ações de saúde, mesmo no modelo normativo, está longe de centrar-se numa racionalidade técnica. E dificilmente, dentro do enfoque eștratégico de planejamento, a epidemiologia (social) terá oportunidade de atuar decisivamente no presente contex to. É flagrante a percepção de que uma linha de epidemiologia que localiza sua crítica essencial quanto à causalidade do processo saúde/doença nas acentuadas desigualdades sócio-econômicas não será aceita pelos setores hegemônicos que procuram manter esta mesma estrutura social e que dispõem do poder de interferir nas decisões quanto às políticas de saúde.

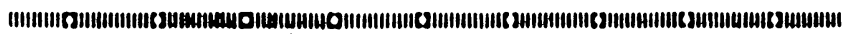

This paper discusses the relationship between the epidemiological tool and the planning methods applied to Public Health activities in Brazil. In order to achieve this, current ideas and trends of these disciplines are briefly reviewed. An analytical framework related to rationality in the planning process is considered. At last, possible reasons for explaining health planning failure in Brazil and the role of Epidemiology within this context are presented.

\section{REFERÊNCIAS BIBLIOGRÄFICAS}

1. ALMEIDA FILHO, N. Problemas e perspectivas atuais da pesquisa epidemiológica em medicina social. In: Textos em Epidemiologia. Brasília SEPLAN/CNPq, 1984.

2. BREILH, J. Epidemiologia; economia, medicina y política. Santo Domingo, Sespas, 1981.

3. CARVAlHeiro, J.R. Processo migratório e disseminação de doenças. Tex tos de Apoio - Ciências Sociais I. PEC/ENSP, ABRASCO, 1983.

4. CHORNY, A. et alii - La epidemılogia, la planificación, la necesidad de atención de la salud y los sistemas de información. Rev. Atención Méd., 2, (3/4): 125-61, dic. 1973/ mar. 1974.

5. CREVENNA, P.B. Algunas consideraciones sobre la evolución del concep to de epidemiologia. Saúde em Debate, 4: 35-8, 1977.

6. DEVER, G.E.A. Holistic health; an epidemiological model for policy analysis. In: Epidemiology in health services management. Rockwell, Aspen publ., 1983.

Caderno de Saúde Pública, R.J., 1(4) :447-456, out/dez, 1985. 
7. FERREIRA, F.W. Planejamento sim e não. Rio de Janeiro, Paz e Terra, 1981.

8. GIORDANI, J.A. La planificación como proceso social; un esquema de análisis. Cuad. Soc. Venez. Planif., (3): 142-9, 1979.

9. KNOX, E.G., ed. Epidemiology in heal th care planning. London, Oxford University Press, 1979.

10. LAST, J., ed. Dictionary of epidemiology. New York, Oxford University Press, 1983.

11. LAURELL, A.C. Algunos problemas teóricos y conceptuales de la epidemiologia social. 1976. mimeo.

12. LEAVELL, H. \& CLARK E.G. Medicina preventiva. Rio de Janeiro. McGraw-Hill, 1978.

13. LILIENFELD, D.E. Definitions of epidemiology. Am J. Epidemiol, 107 (2): 87-90, 1978.

14. MACMAHON, B. \& PUGH, T. Principios y métodos de epidemiologia. México, La Prensa Médica Mexicana, 1975.

15. MIGLIOLI, I. Introdução ao planejamento econômico. Rio de Janeiro, Brasiliense, 1980.

16. MORGENSTERN, H. Uses of ecology analysis in epidemiological research. Am. J. Public. Health, 72 (12): p. 1982.

17. MORRIS, J.N..Uses of Epidemiology. London, Churchill Livingstone, 1975.

18. QUADRA, A.A:F. Viver é resistir. Rio de Janeiro, Achiamé, 1983.

19. SACKETT, D.; HAYNES, R.B. \& TUGWELL, P. Clinical epidemiology; a basic science for clinical medicine. Boston, Little, Brown and Co., 1985.

20. SUSSER, M. Causal thinking in the health sciences. New York, Oxford University Press, 1973.

21. TAMBELlinI, A. Análise dos determinantes das condições de saúde da população brasileira. In: GUIMARÃES, R. org. Saúde e medicina no Brasil. Rio de Janeiro, Graal, 1978.

22. TANNEN, L. Health planning as a regulatory strategy; a discussion of its history and current uses. $J$ Health Serv., 10 (1): $127-9,1985$.

23. TERRIS, M. La revolución epidemiológica y la medicina social. México, Siglo Veintiuno, 1981. 